\title{
A Critique of Stephen Downes' Article, "Learning Objects": A Chinese Perspective
}

Fuhua (Oscar) Lin

This paper by Stephen Downes recommends a way of sharing online teaching/course materials to accelerate course development and make education more cost-effective. His paper is a review of basic information about learning objects (LOs) and includes examples that illustrate such technical terms as XML and TML.

\section{Main Contributions}

Downes makes three main contributions in his article:

1. A definition of learning objects, together with an explanation of why we need learning objects (LOs). Some persuasive examples, using analytic data, are cited to support the need.

2. Presentation of theoretical aspects of object-oriented design and open standards.

3. An elementary discussion of how to author, design, and display learning objects.

\section{Shortcomings}

The paper does not identify several important issues such as: (a) the level of granularity of learning objects, (b) selection and integration of learning objects in an appropriate way to form higher level units of study, (c) training of professors in the use of learning objects, (d) appropriate use of metadata to facilitate composition of higher level units, and (e) the potential of computer agents to facilitate the dynamic composition of personalized lessons. An unorganized aggregate of learning objects simply does not constitute a course. In order to create a properly designed final course, student and instructor interaction must be built in.

To utilize learning objects requires application of educational systems re-engineering, in which many human factors, as well as organizational and managerial issues, 
will arise. For example, there is currently a shortage of appropriate design methodologies for LOs-based electronic learning environments. Without an appropriate design and development methodology it is difficult to estimate the cost of creating high quality metadata for each object as well as the cost of storing and managing the objects themselves. The author skips over the logistics involved in the implementation of LOs but it is exactly at that level that theoretical and practical work needs to be accomplished. Additionally, with the rapid evolution of technology there is danger that LOs that have been created at great cost for some subjects can quickly become outdated.

The LO model reinforces the notion that course development now needs to follow a systems development methodology and needs to governed by something like a systems development life cycle. It is quite clear that courses are much more than a collection of learning objects. Edubox [http://edubox.nl/] from the Open University (Netherlands) is a clear demonstration of this.

In summary, more than learning objects alone are needed in the creation of an electronic learning environment. Some technical issues, (e.g., instructional design theory and agent-based learning system design) must also be addressed in order to implement learning objects on a large scale.

Another dimension that is not addressed in the paper is the implications of learning objects from a wider international perspective. I will focus on that perspective for the remainder of my review.

\section{An International Perspective on Learning Objects}

Learning object technology is not just another North American fad. In Europe, ARIADNE, PROMETEUS, and CEN/ISS are conducting research, and international cooperation and application related to learning objects. For example, ARIADNE is a research and technology development (RTD) project pertaining to the "Telematics for Education and Training" sector of the 4th Framework Program for Research and Development of the European Union. The project focuses on the development of tools and methodologies for producing, managing and reusing computer-based pedagogical elements and telematics-supported training curricula. Validation of the project's concepts is currently taking place in various academic and corporate sites across Europe.

The International Standardization Organization (ISO) has set up a JTC1/SC36 committee, charged to develop International Information Technology Standards in the areas of Learning, Education, and Training [http://jtc1sc36.org/].

In China, the Ministry of Education of PRC has formulated Distance Learning Technology Standards (DLTS) (using XML technology), similar to IEEE LTCS. The objectives of this work are to avoid waste of effort and money due to 
redundancy and low quality and to facilitate international communication and cooperation.

\section{Advantages of learning objects for distance edu- cation in the global market}

Learning objects are not only a technology but also a vision in which the creation of instruction can be automated. LOs computer programs can be devised to inspect, select, and assemble in order to instantiate instructional designs. Grasping the potential of LOs, many institutions of higher education have invested in campus-wide instructional systems based on LO approach to courseware design and delivery. A growing number of international virtual universities and courses are becoming available.

Most applications of LOs are distributed as XML files that can be translated into various languages of the world, such as English, Chinese, French, and so forth and displayed using a wide variety of hardware and software combination. A global LOs market is being formed, similar to supply chains in global manufacturing. In the future, more and more international virtual universities and courses will become available. As soon as China enters into the World Trade Organization (WTO), some universities outside China will enter into the education market in China and Chinese universities may offer courses to students overseas.

\section{Weaknesses of learning objects for distance edu- cation outside North America.}

Online course materials written in one language will not be easily usable internationally because they have to be translated into other languages first. Downes points out that when they use LOs, course developers do not need to prepare all course materials from scratch; hence they can produce courses more economically. This will be the case in the long run, but not in the short run. There will be tremendous start-up costs in generating an international inventory of viable LOs. 
A Critique of Stephen Downes' Article, "Learning Objects": A Chinese

Perspective

\section{Practicalities of learning objects for distance ed- ucation in the global market}

We can have a standard among universities and colleges sharing the same learning objects. At first this standard can be implemented only within a very limited number of units. For example, Downes mentions a project within six universities. With the successful implementation of standard LOs, resultant heightened understanding of instructional design process, the appropriate ontology, and the construction of distributed (agent-based) learning systems, more universities and colleges in the world will become involved in the implementation effort.

\section{Impracticalities of learning objects for distance education in the global market}

In my view, more attention has to be given to management issues for LOs to catch on. Course materials will be created by many authors in different places around the world, and in different languages. Development of a learning object ontology, creation of metadata for describing objects, development of a system of management, distribution, the translation process, and integration into higherlevel units will incur tremendous start-up costs even if they are small relative to the ongoing costs of education and then compared to the long-term savings.

The feasibility of learning objects design may vary from course to course. For instance, the core common elements of an engineering course can be more easily defined and accepted than that of a humanities course. For subjects such as history or philosophy, we need to consider other important factors in selecting LOs, such as, political systems, economic benefits, culture exclusion and protection. In conclusion, much effort is required in order for learning object technology to become practical on a global scale.

\footnotetext{
Citation Format

Lin, Fuhua (Oscar) (July, 2001) A Critique of Stephen Downes' Article, "Learning Objects": A Chinese Perspective. International Review of Research in Open and Distance Learning: 2, 1. http://www.icaap.org/iuicode?149.2.1.8
} 